\title{
Pacific
}

Journal of

Mathematics

\section{ON SOME PROPERTIES OF EXHAUSTION MAPS BETWEEN BOUNDED DOMAINS}

\author{
Chi Keung Cheung
}

Volume $156 \quad$ No. 1 


\title{
ON SOME PROPERTIES OF EXHAUSTION MAPS BETWEEN BOUNDED DOMAINS
}

\section{Chi-Keung Cheung}

\begin{abstract}
In this paper, we study the properties of exhaustion maps defined on Thullen domains and piecewise smooth intersection domains. In general, "exhaustion between two bounded domains" is a much weaker condition than biholomorphism, but in the above two cases, we are able to show that the two concepts are almost the same. In particular, we generalize Pinchuk's theorems to the case of exhaustion maps in the domains mentioned above.
\end{abstract}

1. Introduction and statement of results. It is well known that two bounded domains in $\mathbb{C}^{n}, n>1$, are rarely biholomorphically equivalent to each other. In 1907, Poincare showed that the ball and the polydisc in $\mathbb{C}^{2}$ were not biholomorphic to each other. This result was strengthened by Henkin [7] and Pinchuk [10] to bounded domains with various boundary regularity and convexity conditions. In particular, they have the following remarkable results:

Theorem A ([10]). Let $D$ be a convex but not strictly pseudoconvex domain in $\mathbb{C}^{n}$ with boundary of class $\mathscr{C}^{2+\varepsilon}, \varepsilon>0$. Then $D$ cannot be biholomorphic to any bounded strictly pseudoconvex domain.

Theorem B $([7,10])$. Let $D$ be a bounded pseudoconvex domain in $\mathbb{C}^{n}$, with piecewise $\mathscr{C}^{2}$ smooth (see the definition below) but not smooth boundary. Then $D$ cannot be biholomorphic to any bounded domain with $\mathscr{C}^{2}$ boundary.

By a domain $D$ with piecewise $\mathscr{C}^{k}$ smooth boundary, we mean that in some neighborhood $U$ of $\bar{D}$, there exist real functions $\rho_{\nu}$ defined on it, where $\nu=1, \ldots, m$, such that

(a) $\partial D \subset \bigcup_{1}^{m} S_{\nu}$, where $S_{\nu}=\left\{z: \rho_{\nu}(z)=0\right\}$;

(b) for any subset $\left\{i_{1}, \ldots, i_{q}\right\} \subset\{1, \ldots, m\}, d \rho_{i_{1}} \wedge \cdots \wedge d \rho_{i_{q}} \neq 0$, at every point where $\rho_{i_{1}}=\cdots=\rho_{i_{q}}=0$.

In this paper, we study the conditions when two domains are "almost equivalent" in the sense of exhaustion as defined below.

Definition. Let $D, G$ be bounded domains in $\mathbb{C}^{n}$. We say that " $G$ can be exhausted by $D$ ", or " $D$ exhausts $G$ ", if for every 
compact $K \subset G$ there is a holomorphic imbedding $F: D \rightarrow G$ such that $F(D) \supset K$. Since $D$ and $G$ are bounded domains, if $D$ exhausts $G$, one can always find a countable collection of exhaustion mappings $\left\{F_{k}: D \rightarrow G\right\}$, where each $F_{k}$ is a holomorphic imbedding and for any compact set $K \subset G$, there exists a number $N>0$ such that $F_{k}(D) \supset K$, for all $K>N$.

Obviously, "exhaustion between two bounded domains" is a much weaker condition than biholomorphism. If $D$ and $G$ are biholomorphic, then they can be exhausted by each other, but the converse is false. The above notion of "exhaustion" was studied extensively in [1], [2], [3]. In some situations, exhaustion is almost the same as biholomorphism. For example in [1], it is shown that the unit ball cannot exhaust the polydisc, or more generally, if $D$ exhausts $G$ and $D$ is a homogeneous bounded domain, then $G$ has to be biholomorphic to $D$ ([1], [2]). In the case when $D$ is a strictly pseudoconvex bounded domain with a $\mathscr{C}^{3}$ boundary, then $G$ is either biholomorphic to $D$ or $B^{n}$, the unit ball in $\mathbb{C}^{n}([2])$.

The purpose of this note is to extend the above results to some weakly pseudoconvex domains and partially generalize Theorems A and $B$ to the case of exhaustion maps.

The first type of weakly pseudoconvex domains we study is the Thullen domain, defined by $D_{p}=\left\{\left(z_{1}, z_{2}\right):\left|z_{1}\right|^{2}+\left|z_{2}\right|^{2 / p}<1\right\}$, where $0<p<1$. These domains interpolate between the unit ball $B^{2}$ and the polydisc $B^{1} \times B^{1}$, and the boundary points $\left|z_{1}\right|=1$ are $\mathscr{C}^{2}$ weakly pseudoconvex. Moreover, it is well known that the Thullen domain is never biholomorphic to the ball. We have

Theorem 1. A Thullen domain cannot be exhausted by another Thullen domain, unless they are biholomorphic to each other.

Since $D_{p}$ is a convex domain with boundary of class $\mathscr{C}^{2+\varepsilon}$, by Theorem A, it cannot be biholomorphic to any bounded strictly pseudoconvex domain. For the exhaustion analogue, we have

Theorem 2. A Thullen domain cannot exhaust any bounded strictly pseudoconvex domain unless the latter is biholomorphic to the unit ball.

Theorems 1 and 2 can easily generalize to domains of the form

$$
\left\{\left(z_{1}, z_{2}, \ldots, z_{n}\right):\left|z_{1}\right|^{2}+\left|z_{2}\right|^{2}+\cdots+\left|z_{n-1}\right|^{2}+\left|z_{n}\right|^{2 / p}<1\right\}
$$

in $\mathbb{C}^{n}$. However we would like to remark here that these theorems 
cannot be improved to the generalized Thullen domains:

$$
\left\{\left(z_{1}, z_{2}, \ldots, z_{n}\right):\left|z_{1}\right|^{2}+\left|z_{2}\right|^{2 / p_{2}}+\cdots+\left|z_{n-1}\right|^{2 / p_{n-1}}+\left|z_{n}\right|^{2 / p_{n}}<1\right\},
$$

since, in general, the domain $\left\{\left|z_{1}\right|^{2}+\left|z_{2}\right|^{2 / p_{2}}+\cdots+\left|z_{n-1}\right|^{2 / p_{n-1}}+\right.$ $\left|z_{n}\right|^{\left.2 / p_{n}<1\right\}}$ can exhaust the domain $\left\{\left|z_{1}\right|^{2}+\left|z_{2}\right|^{2}+\cdots+\left|z_{n-1}\right|^{2}+\right.$ $\left.\left|z_{n}\right|^{2 / p}<1\right\}$, even though they are not mutually biholomorphic.

We would like to point out here that any Thullen domain can exhaust the unit ball. For example, consider

$$
D_{0.5}=\left\{\left(z_{1}, z_{2}\right):\left|z_{1}\right|^{2}+\left|z_{2}\right|^{4}<1\right\}
$$

with the family of mappings $F_{k}: D_{0.5} \rightarrow B^{2}$ defined by

$$
F_{k}\left(z_{1}, z_{2}\right)=\left(\frac{\left(z_{2}-1\right)+1 / k}{\left(z_{2}-1\right)-1 / k}, \frac{\sqrt{-1 / k} z_{1}}{\left(z_{2}-1\right)-1 / k}\right), \quad k \geq 2 .
$$

It is easy to check that $D$ exhausts the unit ball through $\left\{F_{k}\right\}$.

The second type of weakly pseudoconvex domains we consider are piecewise $\mathscr{C}^{2}$ smooth intersection domains, that is, those obtained from the intersection of two or more strongly pseudoconvex domains in $\mathbb{C}^{n}$ with $\mathscr{C}^{2}$ boundaries. Precisely speaking, such domains can be represented as

$$
D=\left\{z \in \mathbb{C}^{n}: \rho_{1}(z)<0, \ldots, \rho_{k}(z)<0\right\}, \quad k>1,
$$

where:

(a) for each $j=1, \ldots, k$, the inequality $\rho_{j}(z)<0$ defines a strongly pseudoconvex domain with a $\mathscr{C}^{2}$ boundary, and

(b) the defining functions satisfy $d \rho_{i_{1}} \wedge \cdots \wedge d \rho_{i_{q}} \neq 0(q \leq k)$, at every point where $\rho_{i_{1}}=\cdots=\rho_{i_{q}}=0$.

Obviously, a bounded piecewise $\mathscr{C}^{2}$ smooth intersection domain satisfies the conditions of Theorem B, so it cannot be biholomorphic to any bounded domain with $\mathscr{C}^{2}$ smooth boundary. We are able to generalize this to

THEOREM 3. Let $D$ be a bounded piecewise $\mathscr{C}^{2}$ smooth intersection domain in $\mathbb{C}^{n}$. Then $D$ cannot exhaust any bounded domain in $\mathbb{C}^{n}$ with smooth $\mathscr{C}^{2}$ boundary unless the latter is biholomorphic to the unit ball.

The proofs of these three theorems are similar. They are divided into two steps. The first step is to use the rescaling method introduced 
in [5], [8] and [11] to construct a suitable limiting domain, with the desirable exhaustion property. The second step is to use the normal families argument [13] to obtain the biholomorphic map from the exhaustion maps.

We would like to thank K.-T. Kim, W. K. To and H. Wu for many helpful discussions.

2. Lemmas on exhaustion maps between bounded domains. First we would like to recall the definition of Hausdorff convergence. Given a set $S$ in $\mathbb{C}^{n}$, the Hausdorff distance from a point $p$ to $S$ is defined as

$$
d(p, S)=\inf _{q \in S}\{\text { Euclidean distance between } p \text { and } q\},
$$

and the Hausdorff distance between two sets $S_{1}, S_{2}$ in $\mathbb{C}^{n}$ is defined as

$$
d\left(S_{1}, S_{2}\right)=\max \left\{\sup _{p \in S_{2}} d\left(p, S_{1}\right), \sup _{q \in S_{1}} d\left(q, S_{2}\right)\right\} .
$$

The Hausdorff $R$-seminorm is

$$
d_{R}\left(S_{1}, S_{2}\right)=d\left(S_{1} \cap B(0, R), S_{2} \cap B(0, R)\right)
$$

and we say $\left\{S_{i}\right\}$ converges to $S$ in the sense of Hausdorff if

$$
\forall R>>0, \quad d_{R}\left(S_{i}, S\right) \rightarrow 0 .
$$

In the language of Hausdorff convergence, the notion of $D$ exhausts $G$ through mappings $\left\{f_{k}\right\}$ implies that $f_{k}(D)$ converges to $G$.

We need a lemma to transform the exhaustion properties into biholomorphism. It is a simple generalization of Lemma 1.1 in [2] and also Lemma 1 in [6].

Lemma 1. Let $D$ and $G$ be two bounded domains in $\mathbb{C}^{n}$. Let $\left\{C_{k}\right\}$ be a sequence of open sets in $\mathbb{C}^{n}$ whose closure converges to the closure of $D$ in the sense of Hausdorff convergence. Suppose there exists a sequence of mappings $\left\{F_{k}: C_{k} \rightarrow G\right\}$, satisfying the following conditions:

(1) $F_{k}: C_{k} \rightarrow F_{k}\left(C_{k}\right)$ is biholomorphic for each $k$.

(2) $\left\{F_{k}\left(C_{k}\right)\right\}$ is a sequence of open sets that exhaust $G$ (that is, for any compact $K \subset G$, there exists a number $N$, such that $F_{k}\left(C_{k}\right) \supset K$ for any $k>N)$.

(3) There exists a point $q$ in $G$, such that the set $\left\{F_{k}^{-1}(q)\right\}$ converges to a point $p$ in $D$.

Then $D$ is bilomorphic to $G$. 
Proof. The proof is similar to that in [2]; we will use the standard normal family argument (cf. [3], [4], [10]). Since $D$ is a bounded domain, there exists open sets $\left\{U_{i}\right\}$ such that $U_{1} \subset \subset U_{2} \subset \subset U_{3} \subset \subset$ $\cdots \subset \subset D$. Since $\bar{C}_{k}$ converges to $\bar{D}$, for each $i$, there exists a number $k_{i}$, such that $C_{k} \supset U_{i}$ and $F_{k}$ is well defined on $U_{i}$, if $k>k_{i}$. Since $G$ is a bounded domain, $\left\{\left.F_{k}\right|_{U_{l}}\right\}_{k>k_{i}}$ forms a normal family. Through an induction argument and by passing to a subsequence if necessary, $\left\{F_{k}\right\}$ will converge uniformly on compact sets to a holomorphic map $F: D \rightarrow \bar{G}$. In the other direction, let $S_{k}=F_{k}^{-1}: F_{k}\left(C_{k}\right) \rightarrow D$. Since $D$ is a bounded domain and $G$ is exhausted by $\left\{F_{k}\left(C_{k}\right)\right\}$, the set of mappings $\left\{S_{k}\right\}$ again forms a normal family. Now using a similar argument as above, one can conclude that $\left\{S_{k}\right\}$ will converge uniformly to $S: G \rightarrow \bar{D}$. By Hurwitz's theorem, $F$ and $S$ will either be nonsingular at every point, or their Jacobian determinants are identically zero.

The remaining part of the proof is then exactly the same as that in [2], in which one uses the decreasing property of the Kobayashi metric to show that $F(D)$ and $S(G)$ contain open sets, and hence their Jacobian determinant cannot be identically zero. So the mappings are regular everywhere and we have

$$
F: D \rightarrow G, \quad S: G \rightarrow D, \quad \text { with } F \circ S=\text { identity on } D .
$$

It follows that $D$ and $G$ are biholomorphic to each other. This completes the proof.

In particular, if $D$ exhausts $G$ with a family of exhaustion maps $\left\{F_{k}\right\}$, then the first two conditions of the lemma are satisfied by letting $C_{k}=D$, for all $n$. So we have

LEMMA 2 (Lemma 1.1 in [2]). Let $D, G$ be two bounded domains in $\mathbb{C}^{n}$. Suppose $D$ exhausts $G$ with a family of exhaustion maps $\left\{F_{k}\right\}$, and there exists a point $q$ in $G$ such that the set $\left\{F_{k}^{-1}(q)\right\}$ converges to a point $p$ in $D$. Then $D$ is biholomorphic to $G$.

\section{Proof of theorems.}

Proof of Theorem 1. Let $\left\{f_{k}\right\}$ be a family of exhaustion maps from a Thullen domain $D_{u}=\left\{\left|z_{1}\right|^{2}+\left|z_{2}\right|^{2 / u}<1\right\}$ to another Thullen domain $D_{v}=\left\{\left|z_{1}\right|^{2}+\left|z_{2}\right|^{2 / v}<1\right\}$, where $0<u, v<1$. We want to show that $D_{u}$ is biholomorphic to $D_{v}$. Consider the set $\left\{f_{k}^{-1}(0)\right\}$ in $D_{u}$; there are two cases. 
Case 1. There exists a subsequence of $\left\{f_{k}^{-1}(0)\right\}$ that converges to a point in $D_{u}$. Then by Lemma $2, D_{u}$ is biholomorphic to $D_{v}$.

Case 2. There exists a subsequence of $\left\{f_{k}^{-1}(0)\right\}$ that converges to a point $p \in \partial D_{u}$. Denote such a subsequence by $\left\{\left(r_{j}, s_{j}\right)=f_{j}^{-1}(0)\right\}$. In $D_{u}$, consider the following automorphisms:

$$
\begin{aligned}
g_{j}\left(z_{1}, z_{2}\right)=\left(\frac{\left(z_{1}-r_{j}\right)}{\left(1-\bar{r}_{j} z_{1}\right)}, \frac{\mu\left(1-\left|r_{j}\right|^{2}\right)^{u / 2}}{\left(1-\bar{r}_{j} z_{1}\right)^{u}} z_{2}\right), \\
\text { where } \mu= \begin{cases}\left|s_{j}\right| / s_{j} & \text { if } s_{j} \neq 0, \\
0 & \text { if } s_{j}=0 .\end{cases}
\end{aligned}
$$

Then $g_{j}$ maps $\left(r_{j}, s_{j}\right)$ to $\left(0, w_{j}\right)$, where $w_{j}=\left|s_{j}\right|\left(1-\left|r_{j}\right|^{2}\right)^{-u / 2}>$ 0 . Now $\left\{f_{j} \circ g_{j}^{-1}\right\}$ forms a new family of exhaustion maps from $D_{u}$ to $D_{v}$. Again, we consider the inverse image set of 0 , that is, $\left\{\left(f_{j} \circ g_{j}^{-1}\right)^{-1}(0)=\left(0, w_{j}\right)\right\}$. There are two cases.

Case A. This set is relatively compact in $D_{u}$. Lemma 2 then implies that $D_{u}$ is biholomorphic to $D_{v}$.

Case B. This set converges to $(0,1) \in \partial D_{u}$. In this case, we will apply the scaling technique at the point $(0,1)$, to show that $D_{u}$ has to be biholomorphic to the unit ball.

Consider the translation $F\left(z_{1}, z_{2}\right)=\left(z_{1}, z_{2}-1\right)$. We have

$$
\begin{gathered}
F\left(D_{u}\right)=\left\{\left(z_{1}, z_{2}\right):\left|z_{1}\right|^{2}+\left|z_{2}+1\right|^{2 / u}<1\right\}, \quad F(0,1)=(0,0), \\
F\left(0, w_{j}\right)=\left(0, w_{j}-1\right) .
\end{gathered}
$$

Define the linear scaling map $L_{j}: \mathbb{C}^{2} \rightarrow \mathbb{C}^{2}$ by

$$
L_{j}\left(z_{1}, z_{2}\right)=\left(\frac{1}{\sqrt{\delta_{j}}} z_{1}, \frac{1}{\delta_{j}} z_{2}\right), \quad \text { where } \delta_{j}=1-w_{j}>0 \text {. }
$$

So $\delta_{j} \rightarrow 0^{+}$as $j \rightarrow \infty$, and $L_{j}\left(0, w_{j}-1\right)=(0,-1)$, for every $j$.

For any $R>0$, let $B(0, R)$ denote the Euclidean ball of radius $R$ with center at 0 . Then $L_{j}\left(F\left(D_{u}\right)\right) \cap B(0, R)$ consists of all the points $\left(z_{1}, z_{2}\right)$ in $B(0, R)$ satisfying the inequality

$$
\begin{aligned}
& \left|\sqrt{\delta_{j}} z_{1}\right|^{2}+\left(\left|1+\delta_{j} z_{2}\right|^{2}\right)^{1 / u}<1 \\
& \quad \Leftrightarrow\left|z_{1}\right|^{2}+\frac{\left(\left|1+\delta_{j} z_{2}\right|^{2}\right)^{1 / u}-1}{\delta_{j}}<0 .
\end{aligned}
$$


Since, for each fixed $z_{2}$,

$$
\lim _{j \rightarrow \infty} \frac{\left(\left|1+\delta_{j} z_{2}\right|^{2}\right)^{1 / u}-1}{\delta_{j}}=\frac{2}{u} \operatorname{Re} z_{2},
$$

the set $L_{j}\left(F\left(D_{u}\right)\right) \cap B(0, R)$ converges uniformly on compact sets to the domain $\left\{\left(z_{1}, z_{2}\right):\left|z_{1}\right|^{2}+\frac{2}{u} \operatorname{Re} z_{2}<0\right\} \cap B(0, R)$. So we have $L_{j}\left(F\left(D_{u}\right)\right)$ converges (in the sense of Hausdorff) to the domain, $\left\{\left(z_{1}, z_{2}\right):\left|z_{1}\right|^{2}+\frac{2}{u} \operatorname{Re} z_{2}<0\right\}$, which is biholomorphic to the unit ball $B^{2}$ through a linear fractional transformation $H$, defined by

$$
H\left(z_{1}, z_{2}\right)=\left(\frac{2 z_{1} u}{-\sqrt{-12 z_{2}+\sqrt{-1} u}}, \frac{-2 z_{2}-u}{-2 z_{2}+u}\right) \text {. }
$$

Also since $\operatorname{Re} z_{2}<0$ for every point $\left(z_{1}, z_{2}\right)$ in $L_{j}\left(F\left(D_{u}\right)\right),\{H \circ$ $\left.L_{j} \circ F: D_{u} \rightarrow B^{2}\right\}$ is a biholomorphic embedding and $\left(H \circ L_{j} \circ F\right)\left(D_{u}\right)$ converges (in the sense of Hausdorff) to the unit ball. Now consider the family of mappings

$$
\left\{f_{j} \circ g_{j}^{-1} \circ\left(H \circ L_{j} \circ F\right)^{-1}:\left(H \circ L_{j} \circ F\right)\left(D_{u}\right) \rightarrow D_{v}\right\} .
$$

We note that:

(1) Since each mapping is a biholomorphic embedding, the composition map

$$
f_{j} \circ g_{j}^{-1} \circ\left(H \circ L_{j} \circ F\right)^{-1}:\left(H \circ L_{j} \circ F\right)\left(D_{u}\right) \rightarrow f_{j}\left(D_{u}\right)
$$

is also biholomorphic.

(2) From the given assumption, $\left\{f_{j}\right\}$ is a family of exhaustion maps from $D_{u}$ to $D_{v}$, so $\left\{f_{j}\left(D_{u}\right)\right\}$ forms a sequence of open sets that exhaust $D_{v}$.

(3) By tracing through the defining maps,

$$
\begin{aligned}
\left(f_{j} \circ g_{j}^{-1} \circ\left(H \circ L_{j} \circ F\right)^{-1}\right)^{-1}(0) \\
\quad=H\left(L_{j}\left(F\left(g_{j}\left(f_{j}^{-1}(0)\right)\right)\right)\right)=H\left(L_{j}\left(F\left(g_{j}\left(r_{j}, s_{j}\right)\right)\right)\right) \\
\quad=H\left(L_{j}\left(F\left(0, w_{j}\right)\right)\right)=H\left(L_{j}\left(0, w_{j}-1\right)\right) \\
\quad=H(0,-1)=\left(0, \frac{2-u}{2+u}\right) \in B^{2} .
\end{aligned}
$$

By Lemma $1, D_{v}$ is biholomorphic to $B^{2}$. This contradicts the fact that any Thullen domain cannot be biholomorphic to the unit ball, and hence this case is impossible.

Proof of Theorem 2. Suppose there exists a family of exhaustion maps $\left\{f_{k}\right\}$ from $D_{u}$ to a strictly pseudoconvex domain $G$. Fix a 
point $q$ in $G$. Consider the set $\left\{f_{k}^{-1}(q)\right\}$ as in the proof of Theorem 1. Replacing $D_{v}$ by $G$, and following a similar argument as above, we see that either $D_{u}$ is biholomorphic to $G$ or $G$ is biholomorphic to the unit ball. But $D_{u}$ cannot be biholomorphic to $G$ (Theorem A), so the result follows easily.

The above two theorems can be easily generalized to domains of the form

$$
\left\{\left(z_{1}, z_{2}, \ldots, z_{n}\right):\left|z_{1}\right|^{2}+\left|z_{2}\right|^{2}+\cdots+\left|z_{n-1}\right|^{2}+\left|z_{n}\right|^{2 / p}<1\right\} .
$$

The proofs will remain the same, except one has to replace the automorphism $g$ by composition of automorphisms of the form:

$$
\begin{gathered}
\left(z_{1}, \ldots, z_{n}\right) \rightarrow\left(\frac{z_{1}\left(1-|\eta|^{2}\right)^{1 / 2}}{1-\bar{\eta} z_{i}}, \ldots, \frac{\left(z_{i}-\eta\right)}{1-\bar{\eta} z_{i}}, \ldots,\right. \\
\left.\frac{z_{n-1}\left(1-|\eta|^{2}\right)^{1 / 2}}{1-\bar{\eta} z_{i}}, \frac{\mu\left(1-|\eta|^{2}\right)^{p / 2}}{\left(1-\bar{\eta} z_{i}\right)^{p}} z_{n}\right),
\end{gathered}
$$

in order to map the inverse points $f_{k}^{-1}(0)$ to $\left(0, \ldots, 0, w_{j}\right)$, for some $w_{j}$.

Proof of Theorem 3. Let $D$ be a bounded piecewise $\mathscr{C}^{2}$ smooth intersection domain. By Theorem B, $D$ cannot be biholomorphic to any bounded domain with $\mathscr{C}^{2}$ boundary. Now let us assume that $D$ can exhaust one such domain. Let $\left\{f_{k}\right\}$ be a sequence of exhaustion maps from $D$ to $G$, a bounded domain with $\mathscr{C}^{2}$ boundary. Again, as in the above two proofs, we would like to consider the limit point of the set $\left\{f_{k}^{-1}(q)=p_{k}\right\}$, where $q$ is a fixed point in $G$. Let us denote this limit point by $p$. If $p$ is in $D$, then by Lemma $2, D$ is biholomorphic to $G$, and this will contradict Theorem B. So $p$ has to be on the boundary. Now, denote the set of singular points on the boundary of $D$ by $S_{\partial D}=\{q \in \partial D: \partial D$ is not smooth at $q\}$. Following Kim's work in [9], there are three different ways the sets $\left\{p_{k}\right\}$ can approach $p \in \partial D$.

Case 1: when $p_{k}$ converges to $p \in S_{\partial D}$ non-tangentially to $\partial D$, that is, there exists a constant $C>0$ such that

$$
\frac{\operatorname{dis}\left(p_{k}, \partial D\right)}{\operatorname{dis}\left(p_{k}, p\right)} \geq C, \quad \text { for any } k \text {. }
$$

Case 2: when $p_{k}$ approaches $p$ tangentially to $S_{\partial D}$, that is, there exists a constant $C>0$, such that

$$
\lim _{k \rightarrow \infty} \frac{\operatorname{dis}\left(p_{k}, \partial D\right)}{\operatorname{dis}\left(p_{k}, p\right)}=0 \quad \text { and } \quad \frac{\operatorname{dis}\left(p_{k}, \partial D\right)}{\operatorname{dis}\left(p_{k}, S_{\partial D}\right)} \geq C \quad \text { for any } k
$$


Case 3: when $p \in \partial D \backslash S_{\partial D}$, or when $p_{k}$ converges to $p$ tangentially to $\partial D$ away from $S_{\partial D}$, that is,

$$
\lim _{k \rightarrow \infty} \frac{\operatorname{dis}\left(p_{k}, \partial D\right)}{\operatorname{dis}\left(p_{k}, p\right)}=0 \text { and } \lim _{k \rightarrow \infty} \frac{\operatorname{dis}\left(p_{k}, \partial D\right)}{\operatorname{dis}\left(p_{k}, S_{\partial D}\right)}=0
$$

For each case, we will use the scaling technique to produce exhaustion mappings from $D$ to a new bounded domain, and then use Lemma 1 to conclude that the new bounded domain is biholomorphic to $G$. The scaling method in each case has been studied carefully in [9] while investigating the asymptotic behavior of the curvature. Although in that paper the domains considered are more restrictive, the general argument using the scaling technique can be carried through without any change. (A similar technique in dealing with piecewise smooth domains can also be found in [11].)

In Cases 1 and 2, one can show that there exists $U$, a neighborhood of $p$ in $\mathbb{C}^{n}$, and a sequence of holomorphic mappings $L_{k}: \mathbb{C}^{n} \rightarrow \mathbb{C}^{n}$, such that $L_{k}(D \cap U)$ converges (in the sense of Hausdorff) to a Siegel domain $D(s, n)$ of the form:

$$
\begin{aligned}
& \operatorname{Im} z_{1}>\left|a_{1 s+1} z_{s+1}\right|^{2}+\cdots+\left|a_{1 n} z_{n}\right|^{2}, \\
& \quad \vdots \\
& \operatorname{Im} z_{s}>\left|a_{s s+1} z_{s+1}\right|^{2}+\cdots+\left|a_{s n} z_{n}\right|^{2},
\end{aligned}
$$

for some number $a_{i j}$. Also $L_{k}\left(p_{k}\right) \rightarrow \hat{p}$, where $\hat{p}$ is an interior point in $D(s, n)$. According to [12], $D(s, n)$ is biholomorphic to $\widetilde{D}$, a $\mathscr{C}^{2}$ piecewise smooth pseudoconvex bounded domain in $\mathbb{C}^{n}$, via a linear fractional transformation $T$. Let us consider the family of mappings $\left\{f_{k} \circ\left(T \circ L_{k}\right)^{-1}: T \circ L_{k}(D \cap U) \rightarrow G\right\}$. One can easily see that if $k$ is large enough, $T$ is holomorphic on $L_{k}(D \cap U), T \circ L_{k}(D \cap U)$ converges to $\widetilde{D}$, and also $\left(f_{k} \circ\left(T \circ L_{k}\right)^{-1}\right)^{-1}(q) \rightarrow T(\hat{p}) \in \widetilde{D}$. Now, if we assume for the moment that the sequence of the image sets $\left\{f_{k}(D \cap U)\right\}$ exhaust the domain $G$, then all the conditions in Lemma 1 are satisfied, so we have $\widetilde{D}$ is biholomorphic to $G$; this again contradicts Theorem B.

For the third case, the scaling technique gives a limit domain defined by

$$
\operatorname{Im} z_{1}>\left|b_{2} z_{2}\right|^{2}+\cdots+\left|b_{n} z_{n}\right|^{2},
$$

which is biholomorphic to the unit ball. A similar argument as above shows that it has to be biholomorphic to $G$ and hence $G$ is biholomorphic to the ball, which is exactly the statement of the theorem. 
To complete the proof, we have to show that $(D \cap U)$ exhausts the domain $G$ under $\left\{f_{k}\right\}$. Let $\left\{V_{i}\right\}$ be a sequence of open sets in $G$, with

$$
q \in V_{1} \subset \subset V_{2} \subset \subset \cdots \subset \subset G .
$$

For each fixed $i$, consider the family of mappings $\left\{\left.f_{k}^{-1}\right|_{V_{i}}: V_{i} \rightarrow D\right\}$. Since $V_{i}$ and $D$ are bounded domains, this family of mappings is relatively compact, and we can assume that $f_{k}^{-1}$ converges uniformly on compact sets to a holomorphic map $g: V_{i} \rightarrow \bar{D}$, with $g(q)=p$. Since $p$ is a point on the boundary of $D$, by its definition, there exists a strictly plurisubharmonic function $\rho$ such that $\rho(p)=0$, and $D \cap U \subset\{z \in U: \rho(z)<0\}$. The function $\rho \circ g$ is plurisubharmonic and is nonpositive; since $\rho \circ g(q)=0$, it follows that $\rho \circ g \equiv 0$. But since $\rho$ is strictly plurisubharmonic, one has $g(z)=p \in U$, for all $z$ in $V_{i}$. This implies that $\left\{f_{k}^{-1}\right\}$ converges uniformly to $p$, and hence there exists a $k_{i}>0$, such that if $k>k_{i}$,

$$
f_{k}^{-1}\left[\bar{V}_{i-1}\right] \subset U \Rightarrow \bar{V}_{i-1} \subset f_{k}[U] .
$$

The claim is then proved by following an induction argument. This completes the proof.

Added in proof. After this paper was submitted for publication, it came to the author's attention that some of the results here (Theorems 1 and 2 and the remarks after Theorem 2) had been discovered independently by A. Kodama, S. Krantz and D. Ma (see their preprint "A characterization of generalized complex ellipsoids in $\mathbb{C}^{n}$ and related results"). The author would like to thank Professors Krantz and Kodama for friendly communications on this matter.

\section{REFERENCES}

[1] H. Alexander, Extremal holomorphic imbeddings between ball and polydisc, Proc. Amer. Math. Soc., 68 (1978), 200-202.

[2] B. L. Fridman, Biholomorphic invariants of a hyperbolic manifold, Trans. Amer. Math. Soc., 276 (1983), 685-698.

[3] J. E. Fornaess and Sibony Nessim, Increasing sequences of complex manifolds, Math. Ann., 255 (1981), 351-360.

[4] S. Frankel, Complex geometry of convex domains that cover varieties, Actâ Math., 163 (1989), 104-149.

[5] _ Affine approach to complex geometry, Contemp. Math., 101 (1989), 263286.

[6] I. Graham and $\mathrm{H}$. Wu, Characterizations of the unit ball $B^{n}$ in complex euclidean space, Math. Z., 189 (1985), 449-456. 
[7] G. M. Henkin, An analytic polyhedron is not holomorphically equivalent to a strictly pseudoconvex domain, Soviet Math. Dokl., 14 (1973), 1026-1029.

[8] K. T. Kim, Complete localization of domains with noncompact automorphism groups, Trans. Amer. Math. Soc., 319 (1990), 139-153.

[9] - Asymptotic behavior of the curvature of the Bergman metric of the thin domains, preprint.

[10] S. Pinchuk, Holomorphic inequivalences of some classes of domains in $\mathbb{C}^{n}$, Math. USSR-Sb., 39 (1981), 61-86.

[11] _ Homogeneous domains with piecewise smooth boundaries, Math. Notes, 32 (1983), 849-852.

[12] I. Pyatetskii-Shapiro, Automorphic Functions and the Geometry of Classical Domains, Gordon and Breach Sci. Publ., New York, London, Paris, 1969.

[13] H. Wu, Normal families of holomorphic mappings, Acta Math., 119 (1967), 193-233.

Received May 13, 1991.

UNIVERSITY OF MICHIGAN

ANN ARBOR, MI 48109-1003 



\title{
PACIFIC JOURNAL OF MATHEMATICS
}

Founded by

\author{
E. F. BECKENBACH (1906-1982) F. WolF (1904-1989) \\ EDITORS
}

\author{
V. S. VARADARAJAN \\ (Managing Editor) \\ University of California \\ Los Angeles, CA 90024-1555 \\ vsv@math.ucla.edu \\ Herbert Clemens \\ University of Utah \\ Salt Lake City, UT 84112 \\ clemens@math.utah.edu \\ F. Michael Christ \\ University of California \\ Los Angeles, CA 90024-1555 \\ christ@math.ucla.edu \\ ThOMAs ENRIGHT \\ University of California, San Diego \\ La Jolla, CA 92093 \\ tenright@ucsd.edu
}

\author{
Nicholas ERCOLANI \\ University of Arizona \\ Tucson, AZ 85721 \\ ercolani@math.arizona.edu \\ R. FINN \\ Stanford University \\ Stanford, CA 94305 \\ finn@gauss.stanford.edu \\ VAUGHAN F. R. JONES \\ University of California \\ Berkeley, CA 94720 \\ vfr@math.berkeley.edu \\ Steven KerckhofF \\ Stanford University \\ Stanford, CA 94305 \\ spk@gauss.stanford.edu
}

C. C. Moore

University of California

Berkeley, CA 94720

MaRTin SCHARLEMANN

University of California

Santa Barbara, CA 93106

mgscharl@henri.ucsb.edu

HAROLD STARK

University of California, San Diego

La Jolla, CA 92093

\author{
UNIVERSITY OF ARIZONA \\ UNIVERSITY OF BRITISH COLUMBIA \\ CALIFORNIA INSTITUTE OF TECHNOLOGY \\ UNIVERSITY OF CALIFORNIA \\ MONTANA STATE UNIVERSITY \\ UNIVERSITY OF NEVADA, RENO \\ NEW MEXICO STATE UNIVERSITY \\ OREGON STATE UNIVERSITY
}

\section{SUPPORTING INSTITUTIONS}

UNIVERSITY OF OREGON

UNIVERSITY OF SOUTHERN CALIFORNIA

STANFORD UNIVERSITY

UNIVERSITY OF HAWAII

UNIVERSITY OF TOKYO

UNIVERSITY OF UTAH

WASHINGTON STATE UNIVERSITY

UNIVERSITY OF WASHINGTON 


\section{PACIFIC JOURNAL OF MATHEMATICS}

Volume $156 \quad$ No. $1 \quad$ November 1992

Enveloping algebras of Lie groups with discrete series

Nguyen HuU AnH and VuOng Manh Son

Asymptotic behavior of eigenvalues for a class of pseudodifferential operators on $\mathbf{R}^{n}$

JUNICHI ARAMAKI

A hybrid of theorems of Vinogradov and Piatetski-Shapiro

ANTAL BALOG and JOHN BENJAMIN FRIEDLANDER

Chaos in terms of the map $x \rightarrow \omega(x, f)$

ANDREW MiCHAEL BRUCKNER and JACK GARY CEDER

Local real analytic boundary regularity of an integral solution operator 97 of the $\bar{\partial}$-equation on convex domains

ZHENHUA CHEN

On some properties of exhaustion maps between bounded domains

Chi Keung Cheung

A generalization of maximal functions on compact semisimple Lie groups

HENDRA GUNAWAN

Stability of nonsingular group orbits

CLARK DEAN HORTON

Bordism and regular homotopy of low-dimensional immersions

JOHN FORBES HuGHES

On six-connected finite $H$-spaces

JAMES PEICHENG LiN and Frank WiLliams 